\title{
2nd international symposium on phytochemicals in medicine and food (2-ISPMF)
}

\author{
Jianbo Xiao $\cdot$ Zhenya Yordanova $\cdot$ Ming Miao
}
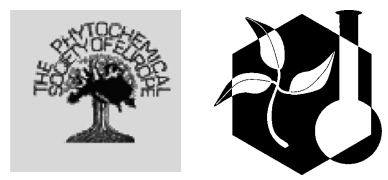

Published online: 3 April 2017

(C) Springer Science+Business Media Dordrecht 2017

The 2nd international symposium on phytochemicals in medicine and food (2-ISPMF) will be held from April 7th to 10th, 2017 in Fuzhou, China. 2-ISPMF is organized jointly by the Phytochemical Society of Europe (PSE), Phytochemical Society of Asia (PSA) and International Society for Chinese Medicine (ISCM). It dedicates to creating a stage for exchanging the latest research results in phytochemicals for food and human health.

\section{J. Xiao ( $₫)$}

College of Food Science, Fujian Agriculture and Forestry University, Fuzhou 350002, Fujian, People's Republic of China

e-mail: jianboxiao@yahoo.com

J. Xiao

Institute of Chinese Medical Sciences, State Key

Laboratory of Quality Research in Chinese Medicine,

University of Macau, Taipa, Macau

\section{Z. Yordanova}

Department of Plant Physiology, Faculty of Biology, Sofia University "St. Kl. Ohridski", 8 Dragan Tzankov Blvd., 1164 Sofia, Bulgaria

e-mail: jiordanova@gmail.com

M. Miao

State Key Laboratory of Food Science and Technology, Jiangnan University, 1800 Lihu Avenue,

Wuxi 214122, Jiangsu, People's Republic of China

e-mail: miaoming@jiangnan.edu.cn
It is jointly organized by Fujian Agriculture and Forestry University, University of Macau, and University of Hong Kong. The local sponsoring institutions contain Kunming University of Science and Technology, Yancheng Institute of Technology, Guiyang Medical University, Nanchang University, Hebei Normal University of Science and Technology, Zhejiang University, Beijing Normal University, Jinan University and so on. Over 250 scientists from 32 counties have registered to attend this conference.

Prof. Baodong Zheng from Fujian Agriculture and Forestry University is the Chairman of local organizing committee. The international organizing committee board of 2-ISPMF consists of Yitao Wang (Macau, Honorary Chairman), Jianbo Xiao (China, Executive Chairman), Yanbo Zhang (Hong Kong, Co-Chairman), Miroslav Strnad (Czech), Yoshinori Asakawa (Japan), Bernhard Hennig (USA), Mei Han (China), Milen I. Georgiev (Bulgaria), Amir Reza Jassbi (Iran), Virginia Lanzotti (Italy), Pinarosa Avato (Italy), Veronique Seidel (UK), Rosa Tundis (Italy). The international scientific committee board is comprised of the representatives from China, Chile, Sweden, Romania, Botswana, Pakistan, USA, Italy, Spain, Austria, Turkey, Algeria, Germany, Thailand, UK, Netherlands, and South Korea.

The international organizing committee and scientific committee board of 2-ISPMF assembled an exciting and diverse program, featuring 14 keynote lectures, 28 invited lectures, 46 short lectures, a 
graduate student forum consisting of 20 oral presentation, and about 100 posters, which dedicate to creating a stage for exchanging the update research results in the phytochemicals for food and human health.

2-ISPMF also has obtained the supports form several international journals including Phytochemistry Reviews (Springer), Food and Chemical Toxicology (Elsevier), Molecules (MDPI), Frontiers in Pharmacology (Frontiers), and Annals of the New York Academy of Sciences (Wiley).

Various nature-origin phytochemicals in medicine and food, such as polyphenols, flavonoids, alkaloids, polysaccharides, stilbenoids, and essential oils, have received an increased attention due to their significant benefits for human beings. Ferns are an important phytogenetic bridge between lower and higher plants. Historically they have been used in many ways by humans, including as ornamental plants, domestic utensils, foods, and in handicrafts. Cao et al. (2017a) summarized bioactive phytochemicals and pharmacology of fern species. The authors pointed out that there is comparatively little information relating to the phytochemicals of ferns and their bioactivities, despite their potential as sources of novel bioactive compounds. They suggested a survey should be carried out to identify rare, traditional and wild fern species used in foods and medicines. Liu et al. (2016) summarized the phytochemicals and pharmacology of Artemisia sieversiana Ehrhart ex willd. Till now, 66 compounds including terpenoids, lignans, flavonoids, steroids and alkaloids were isolated from this plant, which showed antitumor activity, antioxidant activity, and anti-inflammatory activity. However, the efficacy of whole plant was less reported. The phytochemical and pharmacological properties of Trianthema portulacastrum was reviewed by Sukalingam et al. (2017). Based investigation, T. portulacastrum has been found to control oxidative stress and inflammation on the animal model; however, the clinical data are yet inadequate.

Yi et al. (2017) outlined the health-related properties of Citrus flavonoids, including antioxidant activity, anticancer activity, anti-inflammatory activity, and cardiovascular protection. Significant clinical studies with purified Citrus flavonoids should be performed to clarify their benefits in humans. Sharma et al. (2016) reviewed the anticancer potential of andrographolide from Andrographis paniculata and its derivatives. The plausible active pharmacophores included $\alpha$-alkylidene $\gamma$ butyrolactone ring, the conjugated double bond, C-14 hydroxyl or its ester moiety and C8-C17 double bond or epoxy moiety. Behzad et al. (2017) summazied the benefits of phloretin, which showed anticancer activity, antiosteoclastogenic activity, antifungal and antibacterial activities, antiviral activity, anti-inflammatory activity, and so on. Vinayagam et al. (2017) discussed the anti-diabetic properties of dietary phytochemicals. The antidiabetic mechanism included stimulation of insulin secretion, reduction of insulin resistance and increasing of insulin sensitivity, stimulation of glycogenesis and hepatic glycolysis, anti-inflammation and antioxidant, inhibition of digestive enzymes.

Cao et al. (2017b) reviewed the influence of phytochemicals on the biocompatibility of inorganic nanoparticles. Phytochemicals could be used as biofriendly capping agents for green synthesis of nanoparticles with remarkable stability and high therapeutic efficiency. Zhu et al. (2016) overviewed the recent developments in isolation, hydrolysis and characterization for glycosidic aroma precursors in wines, as well as their biological applications.

\section{References}

Behzad S, Sureda A, Barreca D et al (2017) Health effects of phloretin: from chemistry to medicine. Phytochem Rev. doi:10.1007/s11101-017-9500-x

Cao H, Chai TT, Wang X et al (2017a) Phytochemicals from fern species: potential for medicine applications. Phytochem Rev. doi:10.1007/s11101-016-9488-7

Cao Y, Xie Y, Liu L et al (2017b) Influence of phytochemicals on the biocompatibility of inorganic nanoparticles: a stateof-the-art review. Phytochem Rev. doi:10.1007/s11101017-9490-8

Liu SJ, Liao ZX, Tang ZS et al (2016) Phytochemicals and biological activities of Artemisia sieversiana. Phytochem Rev. doi:10.1007/s11101-016-9475-Z

Sharma V, Sharma T, Kaul S et al (2016) Anticancer potential of labdane diterpenoid lactone "andrographolide" and its derivatives: a semi-synthetic approach. Phytochem Rev. doi:10.1007/s11101-016-9478-9

Sukalingam K, Ganesan K, Xu BJ (2017) Trianthema portulacastrum L. (giant pigweed): phytochemistry and pharmacological properties. Phytochem Rev. doi:10.1007/s11101017-9493- 
Vinayagam R, Xiao JB, Xu BJ (2017) An insight into antidiabetic properties of dietary phytochemicals. Phytochem Rev. doi:10.1007/s11101-017-9496-2

Yi LZ, Ma SS, Ren DB (2017) Phytochemistry and bioactivity of Citrus flavonoids: a focus on antioxidant, anti-inflammatory, anticancer and cardiovascular protection activities. Phytochem Rev. doi:10.1007/s11101-017-9497-1
Zhu F, Du B, Ma Y et al (2016) The glycosidic aroma precursors in wine: occurrence, characterization and potential biological applications. Phytochem Rev. doi:10.1007/s11101016-9487-8 